\title{
Diarrhea, CTCAE
}

National Cancer Institute

\section{Source}

National Cancer Institute. Diarrhea, CT CAE. NCI Thesaurus. Code C57788.

A disorder characterized by an increase in frequency and/or loose or watery bowel movements. 